\title{
Efficacy of a leukotriene receptor antagonist for pediatric cedar pollen allergy complicated by asthma
}

\author{
SHIGEMI YOSHIHARA ${ }^{1}$, YUTAKA KIKUCHI $^{2}$, MARI SAITOU $^{2}$, SUSUMU YANAGAWA $^{3}$, \\ NORIKO KANNO $^{1,4}$, HIROSHI IGARASHI ${ }^{5}$, HIRONOBU FUKUDA ${ }^{1,6}$, AKIKO IIMURA ${ }^{7}$, \\ TOSHIO ABE ${ }^{1,8}$, YUMI YAMADA ${ }^{8}$, TAMOTSU ANDOU ${ }^{9}$ and OSAMU ARISAKA ${ }^{1}$
}

\author{
${ }^{1}$ Department of Pediatrics, Dokkyo Medical University, Mibu, Tochigi 321-0293; ${ }^{2}$ Department of Pediatrics, \\ Haga Red Cross Hospital, Mooka, Tochigi 321-4306; ${ }^{3}$ Yanagawa Children's Clinic, Sano, Tochigi 327-0004; ${ }^{4}$ Department of \\ Pediatrics, Nishikata Hospital, Tochigi, Tochigi $322-0601 ;{ }^{5}$ Department of Pediatrics, Shin-Oyama City Hospital, Oyama, \\ Tochigi 323-0827; ${ }^{6}$ Department of Pediatrics, Nasu Red Cross Hospital, Otawara, Tochigi 324-8686; \\ ${ }^{7}$ Himawari Children's Clinic, Utsunomiya, Tochigi 321-0962; ${ }^{8}$ Tsuchiya Children's Hospital, Kuki, \\ Saitama 346-0003; ${ }^{9}$ Andou Children's Clinic, Utsunomiya, Tochigi 321-0923, Japan
}

Received February 23, 2016; Accepted February 24, 2017

DOI: $10.3892 / \mathrm{etm} .2017 .4893$

\begin{abstract}
Leukotriene receptor antagonists (LTRAs) are identified as a monotherapy for asthma and allergic rhinitis; however, their use in children for treatment of these diseases has not been examined. Accordingly, the present study investigated the efficacy of pranlukast dry syrup for children with both pollinosis and asthma. The subjects were children receiving treatment for asthma who were also diagnosed with cedar pollen allergy. Patients were divided into a group that received continuous treatment with pranlukast (group A; $\mathrm{n}=20$ ) and a group that commenced add-on treatment for pollinosis following the onset of symptoms (group $B ; n=20$ ). Patients in group B were randomly allocated to subgroup B1 (add-on treatment with pranlukast dry syrup) or subgroup B2 (add-on treatment with a second-generation antihistamine). In both groups, nasal and ocular symptoms were evaluated every day and recorded in a diary. Exacerbation of nasal obstruction was demonstrated in group B; however, not in group A. There was a significant difference in symptoms observed between the two groups during the late peak pollen period $(\mathrm{P}<0.05)$. The incidence of nasal obstruction (defined as a nasal obstruction score $\geq 3$ or use of a nasal steroid spray) was significantly lower in group A compared with group $\mathrm{B}(\mathrm{P}<0.05)$. The maximum scores for sneezing and nasal obstruction during the late peak of the pollen season were lowest in group A, followed by subgroup B1 and subgroup B2. The present study demonstrated that long-term administration of LTRA for the management of
\end{abstract}

Correspondence to: Dr Shigemi Yoshihara, Department of Pediatrics, Dokkyo Medical University, 880 Kitakobayashi, Mibu, Tochigi 321-0293, Japan

E-mail: shigemi@dokkyomed.ac.jp

Key words: pollen, pranlukast, child, nasal diseases, quality of life asthma may improve nasal symptoms of pollinosis during the pollen season in children with pollinosis and asthma.

\section{Introduction}

The number of pediatric patients with asthma continues to increase each year in Japan (1). Control of asthma in children has improved with the widespread use of inhaled steroids and leukotriene receptor antagonists (LTRAs) to suppress inflammation; however, the response to these medications is not always satisfactory.

Allergic rhinitis (AR) is associated with the occurrence and exacerbation of asthma (2). Although the frequency varies among reports, AR is observed in $70-80 \%$ of children with asthma and the two diseases are closely related $(3,4)$. The incidence of cedar pollen allergy (a seasonal type of AR) has also been increasing, and its prevalence in 5-9-year-old children was demonstrated to be $13.7 \%$, according to a survey performed in 2008 (5). As both diseases affect the airways and exacerbation of nasal symptoms influences asthma symptoms, these two diseases are increasingly viewed from the perspective of the 'one airway, one disease' paradigm (6). Pranlukast dry syrup is an LTRA that is used as a monotherapy for bronchial asthma or AR (7-10); however, its use in children with both diseases has not been examined.

Therefore, the present study was performed to investigate the efficacy of pranlukast dry syrup in children with asthma and nasal symptoms (sneezing, nasal discharge, and nasal congestion) or ocular symptoms (lacrimation, eye-rubbing, and ocular hyperemia) of pollinosis. The study season was divided into 4 periods, namely i) start of the season to early peak period, ii) late peak period, iii) final part of the season, and iv) after the season. The effects of LTRA were assessed by comparing a group of children treated with pranlukast dry syrup as a long-term drug for the management of bronchial asthma, who did not receive any other medication during the pollen season, with another group of children in whom 
bronchial asthma had been controlled with a medication other than pranlukast dry syrup, and who were treated with this LTRA when pollinosis symptoms occurred.

\section{Materials and methods}

Ethics statement. The present study was approved by the Ethics Committee of Dokkyo Medical University Hospital (Mibu, Japan; 26096). Written informed consent was obtained from the parents of the patients enrolled in the study.

Patients. The subjects of the study were children with asthma under long-term treatment at the Pediatric Department of Dokkyo Medical University Hospital and related institutions between May 2008 and March 2012 (demographics presented in Table I). Patients were required to meet the following criteria: i) Aged from 2-14 years; ii) diagnosed with cedar pollen allergy within 2 years prior to enrolment based on detection of eosinophils in nasal discharge or Radio-Allergo-Sorbent Test data; iii) written consent to participate in the present study was provided by the parents; iv) outpatients; and v) the child's asthma was under reasonable control. Asthma symptoms were assessed according to the Japanese Guideline for Childhood Asthma 2012 (1).

The exclusion criteria were as follows: i) Severe asthma, defined as a history of an asthma attack requiring tracheal intubation for ventilation, respiratory arrest, carbon dioxide excess, hypoxic spasm, an asthma attack accompanied by syncope or treatment with oral/parenteral steroids within 4 weeks prior to initiation of the study; ii) respiratory disease other than asthma judged by a physician based on evaluation of the response to the test drugs; iii) hypersensitivity or suspected hypersensitivity to ingredients of the inhaled steroid or LTRA used in the present study; iv) participation in another clinical study; and v) ineligibility for other reasons as judged by the attending physician.

Study groups. The patients were divided into two groups, which either received continuous treatment with pranlukast (group A) or initiated treatment for pollinosis after the onset of symptoms (group B). Patients in group A received pranlukast dry syrup (Ono Pharmaceutical, Co., Ltd., Osaka, Japan) for long-term management of their asthma without addition of further drugs during the pollinosis season. Patients in group B had asthma that was stably controlled by medication other than pranlukast dry syrup and they commenced treatment with pranlukast dry syrup or a second-generation antihistamine (olopatadine hydrochloride (Kyowa Hakko Kirin Co., Ltd., Tokyo, Japan) or loratadine tablets (Bayer Yakuhin, Osaka, Japan) when symptoms of pollinosis developed. The patients in group B were randomly allocated by the envelope method to subgroups receiving add-on treatment with pranlukast dry syrup (subgroup B1) or add-on treatment with a second-generation antihistamine (subgroup B2).

Study treatment. Pranlukast dry syrup was administered orally at a daily dose of $7 \mathrm{mg} / \mathrm{kg}(3.5 \mathrm{mg} / \mathrm{kg}$ twice daily, after breakfast and dinner). Olopatadine hydrochloride was administered orally at a daily dose of $10 \mathrm{mg}(5 \mathrm{mg}$ twice daily, in the morning and before bedtime). Loratadine was administered orally at a daily dose of $10 \mathrm{mg}$ (once a day after a meal).
Table I. Background characteristics of patients receiving continuous treatment with pranlukast dry syrup (group A) and those receiving add-on treatment at the onset of pollinosis (group B).

\begin{tabular}{lccc}
\hline Characteristics & Group A & Group B & P-value \\
\hline Patients (male/female) & $20(11 / 9)$ & $20(15 / 5)$ & \\
Age (years) & $5.3 \pm 0.29$ & $8.7 \pm 0.56$ & $<0.01$ \\
Complications & & & \\
$\quad$ Asthma & $3 / 6 / 11 / 0$ & $8 / 3 / 8 / 1$ & NS \\
Perennial allergic rhinitis & 3 & 6 & NS \\
Allergic conjunctivitis & 2 & 5 & NS \\
Atopic dermatitis & 5 & 9 & NS \\
Others & 1 & 1 & NS \\
\hline
\end{tabular}

antermittent to persistent/mild to persistent/moderate to persistent/severe. Data were analyzed using paired t-tests and chi-square tests and are presented as the mean \pm standard error of the mean where appropriate. NS, not significant.

The following criteria were evaluated in both groups: i) Patient background factors, including age, gender, complications and severity of asthma, and ii) nasal symptoms, including sneezing, runny nose and nasal obstruction, and ocular symptoms, including tears, eye rubbing and hyperemia. Nasal and ocular symptoms were classified into five categories (4, very severe; 3 , severe; 2 , moderate; 1 , mild; 0 , no symptoms) and recorded in a diary every day. The patients or their parents recorded the assessments in the diaries themselves. Assessment was performed three times a day (morning, afternoon, and evening) and the mean value of the three measurements was recorded. Comparison between the groups was performed by using the highest values for each period. The primary endpoint of the present study was to determine the effectiveness of treatment for nasal symptoms, such as sneezing, runny nose, and nasal congestion, based on maximum scores obtained in the peak pollen season.

Treatments for asthma and perennial nasal allergy were not restricted and the drugs used were recorded in the diary. The airborne cedar pollen level in Utsunomiya (Tochigi, Japan) was 3,320 pollen grains $/ \mathrm{cm}^{2}$ in 2012, which was markedly higher than the normal yearly average (Fig. 1; Ministry of Environment; http://kafun.taiki.go.jp/).

Statistical analysis. Statistical analysis was performed using SAS version 9.2 (SAS Institute, Cary, NC, USA). Unpaired t-tests were used for numerical comparisons, while the chi-square test was used for other comparisons, including complications such as asthma, perennial allergic rhinitis, allergic conjuctivitis, and atopic dermatitis. The log-rank test was carried out to compare Kaplan-Meier curves. Data were presented as the mean \pm standard error of the mean. $\mathrm{P}<0.05$ was considered to indicate a statistically significant difference.

\section{Results}

Patient characteristics. A total of 46 patients were enrolled in the present study; however, 6 did not visit the hospital for 


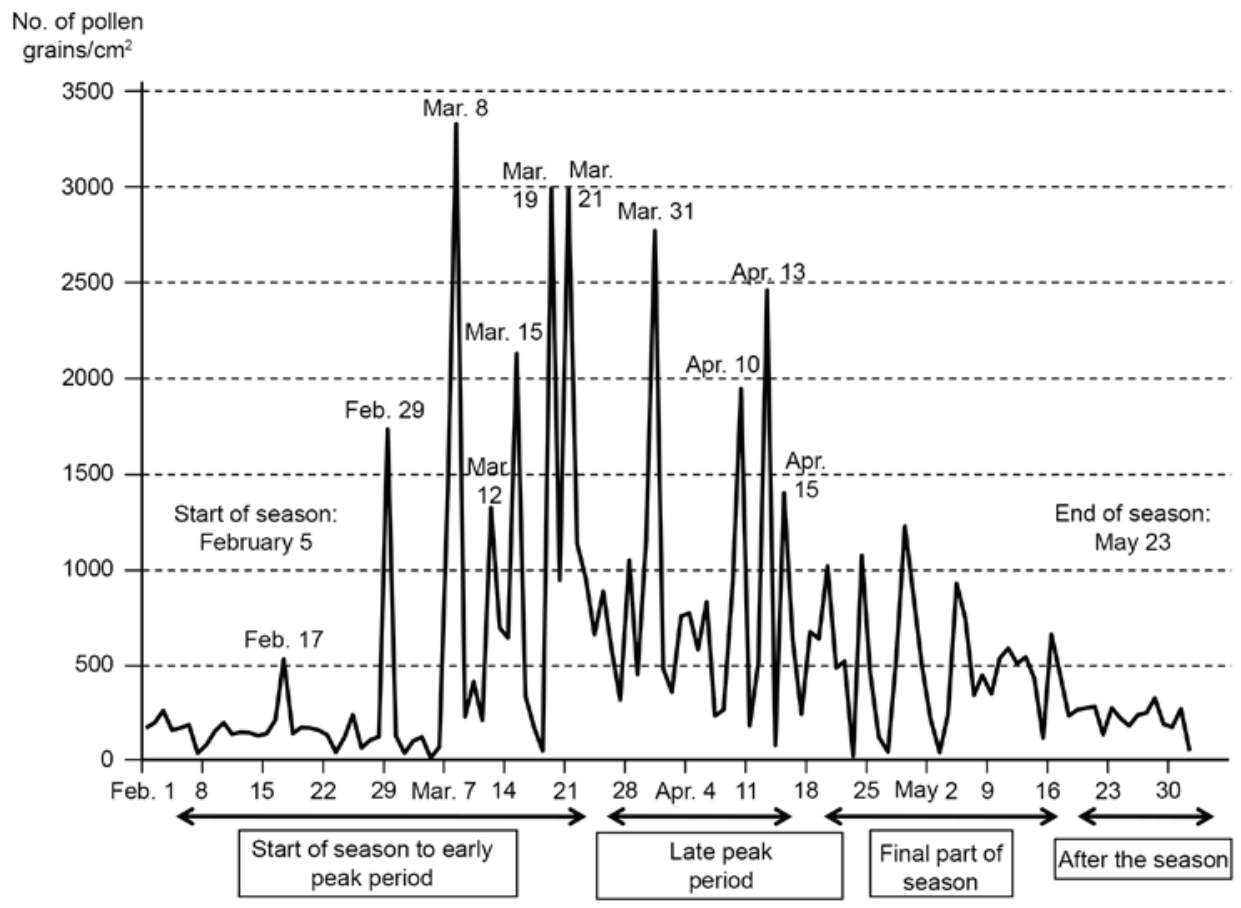

Figure 1. Airborne cedar cypress pollen levels in Utsunomiya (Tochigi, Japan) in 2012 (Based on data from the Ministry of the Environment; http://kafun. taiki.go.jp).

review, leaving 40 patients for analysis. Group A (continuous treatment with pranlukast dry syrup) and group B (add-on treatment for pollinosis) contained 20 patients each. The mean age of group B was significantly higher than group A $(\mathrm{P}<0.01)$. There were no significant differences in the sex ratio or complications, including the severity of asthma, between the two groups (Table I). No adverse events were caused by the drugs administered during the study period.

Nasal symptoms. Changes to nasal symptoms in groups A and B are demonstrated in Fig. 2. Exacerbation of sneezing and runny nose symptoms were observed from the start of the pollen season (February 5th) until after its peak (March 8th) in both groups. Nasal obstruction was also exacerbated in group $\mathrm{B}$; however, this was not observed in group A. There was a significant difference in nasal obstruction between the two groups during the late peak of the pollen season, with group $\mathrm{B}$ demonstrating increased nasal obstruction compared with group $\mathrm{A}(\mathrm{P}<0.05$; Fig. 2).

Ocular symptoms. Changes in ocular symptoms in groups A and B are presented in Fig. 3. Tears did not become significantly more severe in either group throughout the study period. Rubbing of eyes and hyperemia worsened from the start of the pollen season until after its peak in group B; however, this was not observed in group A (Fig. 3).

Nasal obstruction. Changes in the incidence of nasal obstruction (defined as a nasal obstruction score of $\geq 3$ or use of a nasal steroid spray) were compared by. As a result, the incidence of nasal obstruction was significantly lower in group A compared with group B $(\mathrm{P}<0.05$; Fig. 4).

As there was a significant difference in nasal obstruction between groups A and B during the late peak of the pollen season, this period was examined more closely by dividing group B into patients receiving add-on treatment with pranlukast dry syrup (subgroup B1) and those receiving an add-on second-generation antihistamine treatment (subgroup B2).

Stratified analyses. Stratified analyses of the maximum scores for three symptoms were performed. During the late peak period, scores for sneezing and nasal obstruction were lowest in group A, followed by subgroup B1 and subgroup B2, with significant differences observed between the three groups for sneezing and nasal obstruction $(\mathrm{P}=0.009$ and $\mathrm{P}=0.005$, respectively; Fig. 5).

\section{Discussion}

The prevalence of pediatric asthma in Japan was reported to be 4-18\% in 2012 and has been increasing each year (1). Despite this, widespread acceptance of the asthma management guidelines and the accompanying increased use of LTRAs and inhaled steroids has reduced hospitalization of children with asthma and improved control of the condition. However, children with asthma complicated by allergic diseases remain difficult to treat. A survey performed in 2008 demonstrated that the incidence of AR in children was $\sim$ twice as high as it had been 10 years earlier (5). AR is associated with the onset and exacerbation of asthma, suggesting that improvement of nasal symptoms is important to improve control of asthma symptoms.

LTRAs are used as basal or add-on drugs for long-term management of asthma in children (1). In addition, LTRAs are recommended as first-line treatment for AR with nasal obstruction as the primary complaint (5). Early initiation of LTRA treatment prior to the pollen season is reported to be effective (11). Pranlukast dry syrup is an LTRA that suppresses 

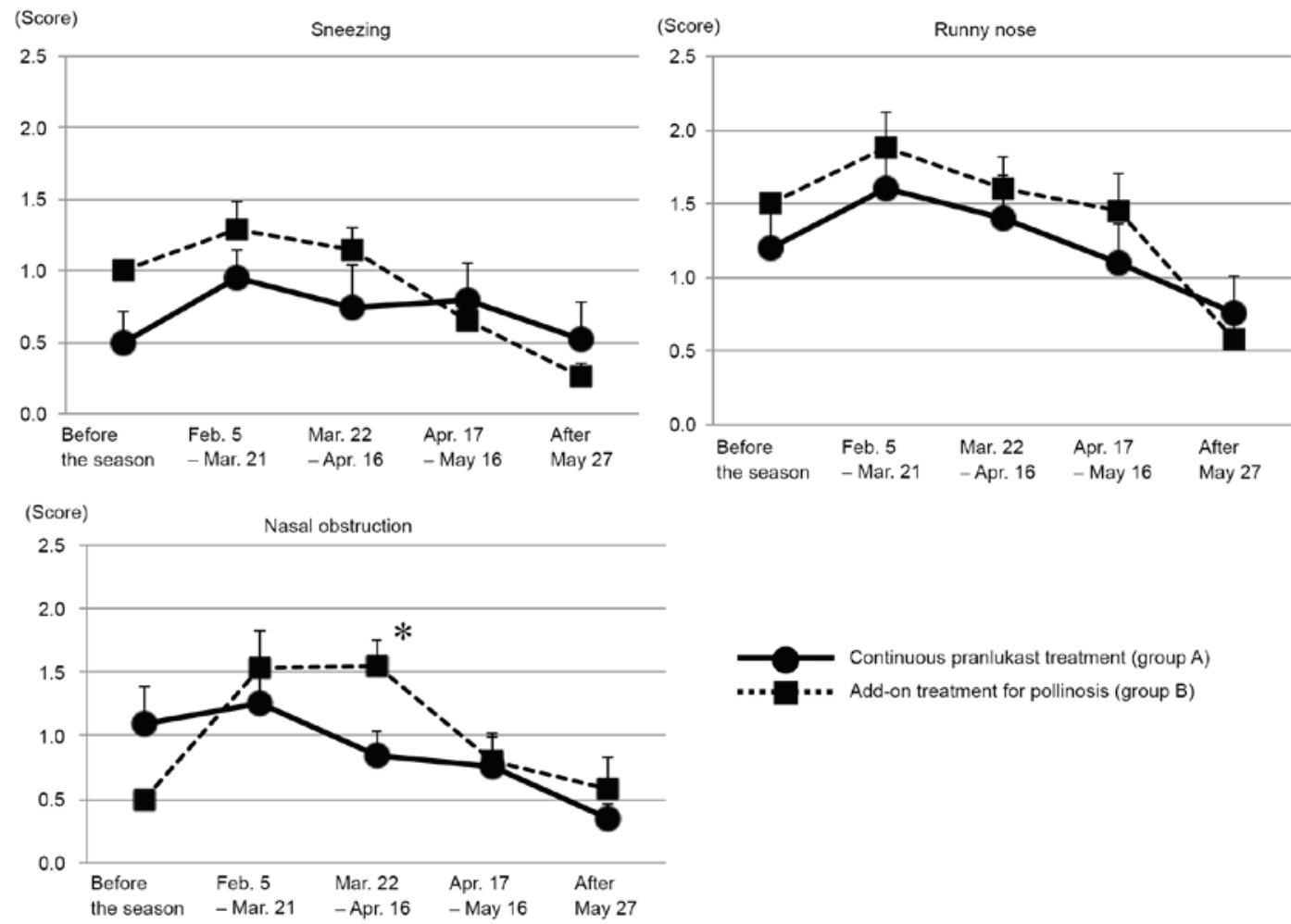

Figure 2. Maximum scores from patient diaries for changes in nasal symptoms demonstrated in groups A and B before, during and after the pollen season. Data were analyzed using paired t-tests and are presented as the mean \pm standard error of the mean. ${ }^{*} \mathrm{P}<0.05$ vs. group A for the period between March 22 and April 16.
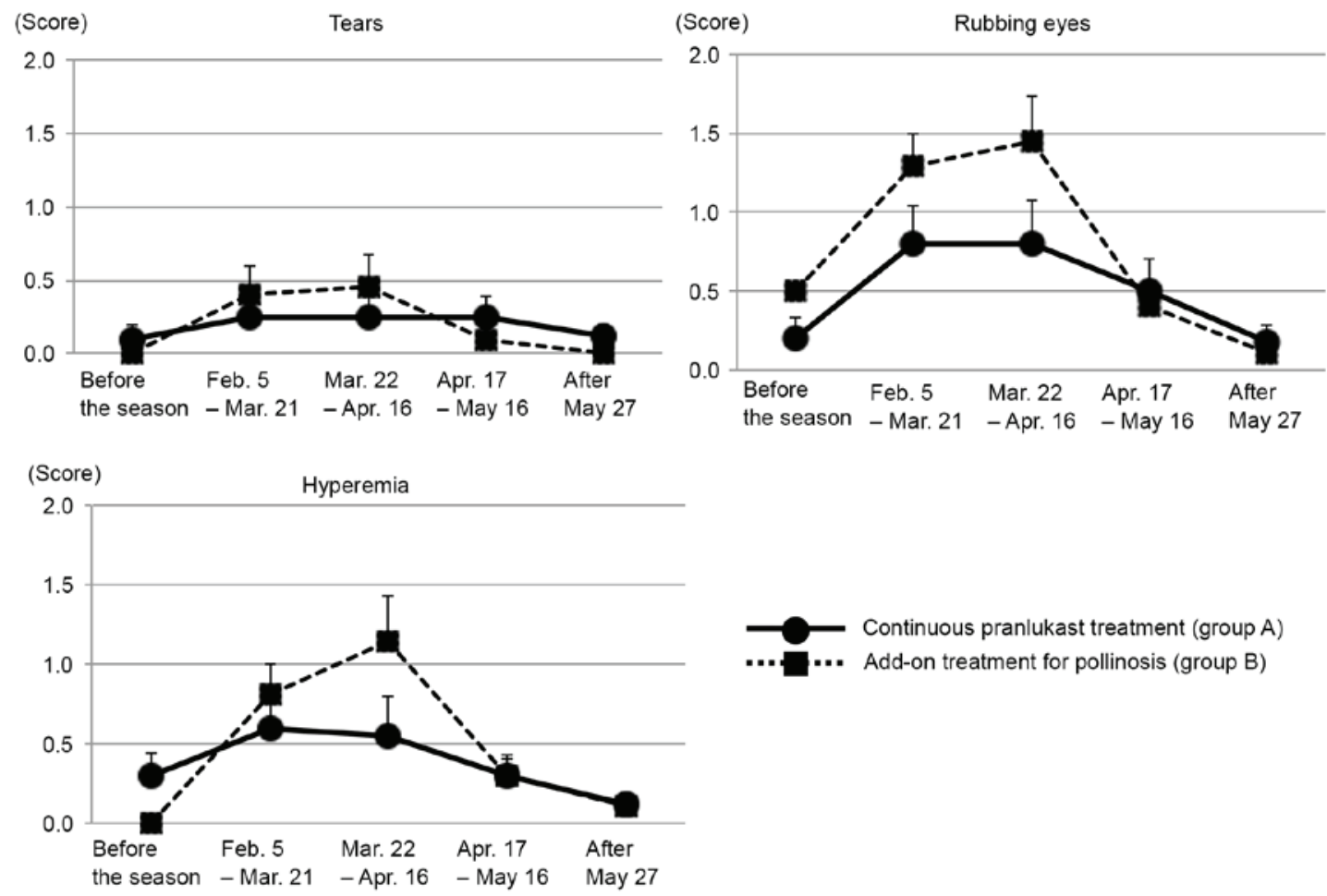

Figure 3. Maximum scores from patient diaries for changes in ocular symptoms demonstrated in groups A and B before, during and after the pollen season. Data were analyzed using paired t-tests and are presented as the mean \pm standard error of the mean.

inflammation of the airway and nasal mucosa by selectively blocking the action of leukotriene produced by mast cells and eosinophils $(12,13)$. In the present study, the efficacy of this drug for children with asthma complicated by cedar pollen allergy was investigated. In children receiving long-term treatment with pranlukast dry syrup for asthma, exacerbation 


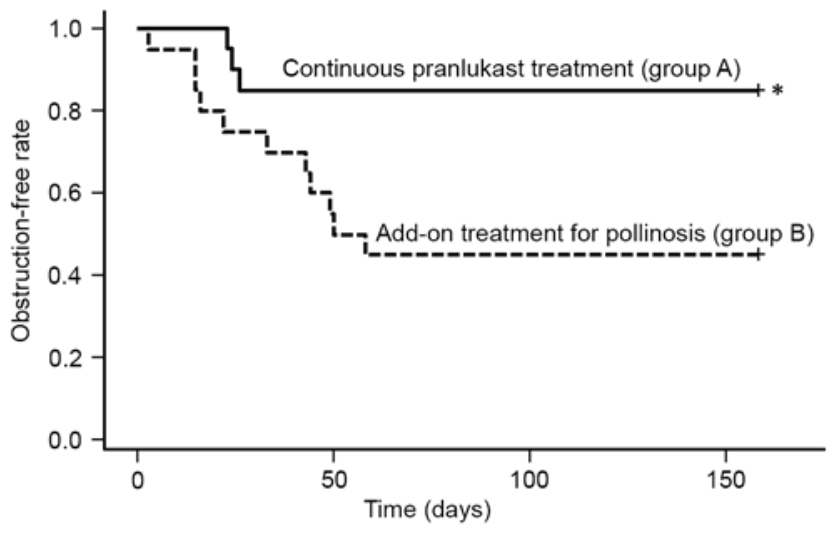

Figure 4. Exacerbation or improvement of nasal obstruction in groups A and B. Kaplan-Meier curves were constructed for nasal obstruction based on a nasal obstruction score $\geq 3$ or the use of steroids. Data were assessed using the log-rank test. ${ }^{*} \mathrm{P}<0.05$ vs. group $\mathrm{B} .+$, discontinuation.

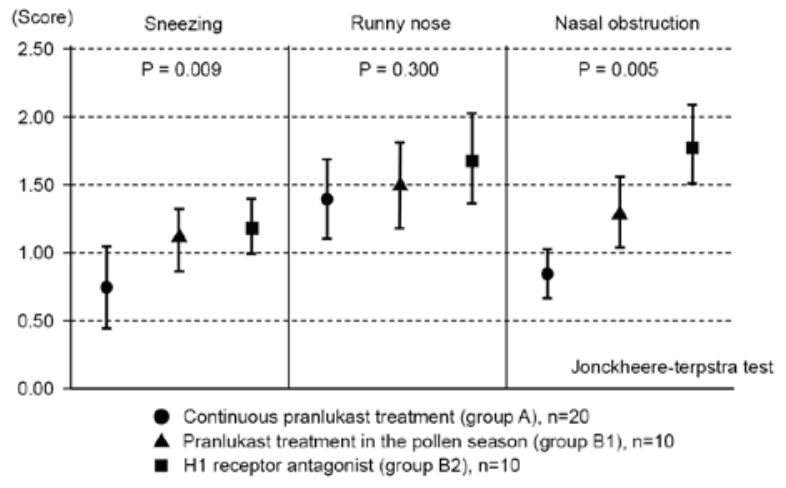

Figure 5. Stratified analysis of the maximum scores for three nasal symptoms during the late peak pollen season. Patients with add-on treatment for pollinosis (group B) were divided into those receiving add-on pranlukast therapy (group B1) and those receiving add-on H1 receptor agonist therapy (group B2). Nasal symptom scores in the late peak period were compared between the two subgroups. Data are presented as the mean \pm standard error of the mean.

of nasal symptoms during the pollen season was reduced and nasal obstruction was significantly decreased in the late peak period compared with children who received add-on therapy with pranlukast dry syrup at the onset of pollinosis symptoms. This difference persisted throughout the pollinosis season.

Release of a small amount of pollen prior to the main pollen season stimulates the nasal mucosa and induces hypersensitivity in patients with pollinosis, a process referred to as priming (14). A previous study performed a pollen challenge test in children with rhinitis and the results demonstrated that the level of eosinophil cationic protein in the nasal discharge was significantly lower in those treated with pranlukast dry syrup than in those receiving placebo (9). In the present study, continuous administration of pranlukast dry syrup may have suppressed nasal symptoms in the peak pollen season by regulating the priming process through inhibition of eosinophilic inflammation. In patients who received add-on pranlukast after the onset of pollinosis symptoms, inflammation of the nasal mucosa may have been controlled early, resulting in reduced nasal obstruction compared with those who received a second-generation antihistamine.
During the pollen season, nasal symptoms impair the quality of life (QOL) of children with cedar pollen allergy. Among these symptoms, nasal obstruction is the most difficult to treat and has a strong impact on QOL (15). Furthermore, exacerbation of nasal symptoms is closely related to aggravation of asthma $(16,17)$. Therefore, alleviation of nasal obstruction by continuous administration of pranlukast dry syrup may improve QOL and asthma control. Initiation of treatment with pranlukast dry syrup after the start of the pollen season may also be useful. Efficacy of pranlukast dry syrup for pollinosis in children has previously been reported on the basis of pollen challenge testing and in patients on long-term administration (up to 20 weeks) of pranlukast dry syrup) $(8,18)$; however, the clinical benefits have not previously been described.

Regarding limitations of the present study, children assessed symptoms themselves or their guardians performed assessment if the children were unable to. There are no reports on the validity of guardians assessing the nasal symptoms of their pre-school children, although this is common in clinical research involving children with rhinitis. However, assessment of objective findings may have provided clearer results and this issue should be considered in future investigations.

A previous comparative study of patients with similar characteristics and symptoms demonstrated that LTRA therapy markedly improved nasal congestion compared with antihistamines, and LTRAs also demonstrated effectiveness against sneezing and runny nose symptoms (10). However, the present study examined the extent to which aggravation of symptoms could be avoided during the peak pollen season in patients receiving continuous treatment, which means that obtaining a clear-cut result may have been unlikely.

In conclusion, the present study demonstrated that long-term LTRA administration for management of asthma may improve nasal symptoms of pollinosis during the pollen season in children with both conditions. However, this was only a pilot study, thus the efficacy of LTRA therapy for children with asthma and AR requires further study in a larger number of patients.

\section{Acknowledgements}

The present study was financially supported by the Japan Allergy Foundation.

\section{References}

1. Hamasaki Y, Kohno Y, Ebisawa M, Kondo N, Nishima S, Nishimuta T, Morikawa A, Aihara Y, Akasawa A, Adachi Y, et al: Japanese pediatric guidelines for the treatment and management of bronchial asthma 2012. Pediatr Int 56: 441-450, 2014.

2. Burgess JA, Walters EH, Byrnes GB, Matheson MC Jenkins MA, Wharton CL, Johns DP, Abramson MJ, Hopper JL and Dharmage SC: Childhood allergic rhinitis predicts asthma incidence and persistence to middle age: A longitudinal study. J Allergy Clin Immunol 120: 863-869, 2007.

3. Masuda S, Fujisawa T, Katsumata H, Atsuta J and Iguchi K: High prevalence and young onset of allergic rhinitis in children with bronchial asthma. Pediatr Allergy Immunol 19: 517-522, 2008.

4. Higuchi O, Adachi Y, Itazawa T, Ito Y, Yoshida K, Ohya Y, Odajima H, Akasawa A and Miyawaki T: Rhinitis has an association with asthma in school children. Am J Rhinol Allergy 27: e22-e25, 2013. 
5. Okubo K, Kurono Y, Fujieda S, Ogino S, Uchio E, Odajima H, Takenaka $\mathrm{H}$ and Baba K; Japanese Society of Allergology: Japanese guideline for allergic rhinitis. Allergol Int 60: 171-189, 2011.

6. Tsilochristou OA, Douladiris N, Makris M and Papadopoulos NG: Pediatric allergic rhinitis and asthma: Can the march be halted? Paediatr Drugs 15: 431-440, 2013.

7. Nishima S, Furusho K, Morikawa A, Mochizuki H, Akasaka T, Sugimoto H, Ikebe T, Nishimuta T, Sekine K, Yamada M, et al: Pranlukast inhibits exercised-induced bronchospasm in asthmatic children: A randomized, multicenter, double-blind, placebo-controlled two-period crossover trial. Pediatr Asthma Allergy Immunol 18: 5-11, 2005.

8. Wakabayashi K, Hashiguchi K, Kanzaki S, Fujioka M, Tanaka N, Kawashima K, Suematsu K, Yamamotoya H, Iwasaki M, Gotoh M, et al: Pranlukast dry syrup inhibits symptoms of Japanese cedar pollinosis in children using OHIO Chamber. Allergy Asthma Proc 33: 102-109, 2012.

9. Gotoh M, Okubo K, Hashiguchi K, Wakabayashi K, Kanzaki S, Tanaka N, Fujioka M, Kawashima K, Suematsu K, Sasaki K, et al: Noninvasive biological evaluation of response to pranlukast treatment in pediatric patients with Japanese cedar pollinosis. Allergy Asthma Proc 33: 459-466, 2012.

10. Yoshihara S: Pranlukast hydrate in the treatment of pediatric bronchial asthma. Pediatr Health Med Ther 4: 55-63, 2013.

11. Gotoh M, Suzuki H and Okubo K: Delay of onset of symptoms of Japanese cedar pollinosis by treatment with a leukotriene receptor antagonist. Allergol Int 60: 483-489, 2011.
12. Obata T, Okada Y, Motoishi M, Nakagawa N, Terawaki T and Aishita H: In vitro antagonism of ONO-1078, a newly developed anti-asthma agent, against peptide leukotrienes in isolated guinea pig tissues. Jpn J Pharmacol 60: 227-237, 1992.

13. Fujita M, Yonetomi Y, Shimouchi K, Takeda H, Aze Y, Kawabata $\mathrm{K}$ and Ohno H: Involvement of cysteinyl leukotrienes in biphasic increase of nasal airway resistance of antigen-induced rhinitis in guinea pigs. Eur J Pharmacol 369: 349-356, 1999.

14. Juliusson S and Bende M: Priming effect of a birch pollen season studied with laser Doppler flowmetry in patients with allergic rhinitis. Clin Allergy 18: 615-618, 1988.

15. Gotoh M, Yuta A, Okano M, Ohta N, Matsubara A and Okubo K: Severity assessment of Japanese cedar pollinosis using the practical guideline for the management of allergic rhinitis in Japan and the allergic rhinitis and its impact on asthma guideline. Allergol Int 62: 181-189, 2013.

16. Yamauchi K, Tamura G, Akasaka T, Chiba T, Honda K, Kishi M, Kobayashi H, Kuronuma T, Matsubara A, Morikawa T, et al: Analysis of the comorbidity of bronchial asthma and allergic rhinitis by questionnaire in 10,009 patients. Allergol Int 58: 55-61, 2009.

17. Kim CW, Lee CW, Hur GY, Ye YM and Park HS; CARINA Study Group: Evaluation and control of allergic rhinitis in adult patients with asthma (CARINA) in Korea. Korean J Asthma Allergy Clin Immunol 27: 248-256, 2007.

18. Konno A, Yamamotoya $\mathrm{H}$ and Okubo $\mathrm{K}$ : Initial treatment using pranlukast dry syrup in children with perennial allergic rhinitis and Japanese cedar pollinosis. Allergology Immunol 20: 280-289, 2013 (In Japanese). 